\title{
Des universités autonomes
}

La réponse japonaise aux défis de l'enseignement supérieur

Autonomous universities. The Japanese answer to the challenges of higher education

Universidades autónomas. La respuesta japonesa a los desafíos de la enseñanza superior

Jun Oba

\section{OpenEdition \\ Journals}

Édition électronique

URL : http://journals.openedition.org/ries/271

DOI : $10.4000 /$ ries. 271

ISSN : 2261-4265

Éditeur

Centre international d'études pédagogiques

Édition imprimée

Date de publication : 1 septembre 2007

Pagination : 135-144

ISBN : 978-2-85420-570-1

ISSN : $1254-4590$

\section{Référence électronique}

Jun Oba, "Des universités autonomes », Revue internationale d'éducation de Sèvres [En ligne], 45 | septembre 2007, mis en ligne le 23 juin 2011, consulté le 21 avril 2019. URL : http:// journals.openedition.org/ries/271; DOI : 10.4000/ries.271 


\section{Des universités autonomes}

\section{La réponse japonaise aux défis de l'enseignement supérieur}

\section{Jun Oba}

Le système d'enseignement supérieur moderne japonais a vu le jour dans la deuxième moitié du XIX ${ }^{\mathrm{e}}$ siècle, et toutes les premières universités ont été organisées sur le modèle universitaire humboldtien. Aujourd'hui, l'enseignement supérieur est assuré essentiellement par deux types d'institutions : Daigaku (université) et Tanki-daigaku (université à cycle court). Ces institutions sont hiérarchisées en fonction de leur origine, localité, réputation, etc., et cette hiérarchisation n'a jamais été fondamentalement remise en cause. L'État a privilégié dans les investissements financiers le secteur national, mais c'est le secteur privé qui accueillent les trois quarts des étudiants. Un numerus clausus, déterminé par l'État, est appliqué à toutes les études universitaires (cycle court inclus), et l'accès à ces institutions est conditionné par un concours d'entrée, organisé sous la responsabilité de chaque établissement. Depuis le milieu des années 1990, le nombre d'étudiants inscrits à ces deux types d'institutions stagne autour de trois millions (3 061404 en 2006), mais les nouveaux entrants sont en diminution depuis 1994. Après une stagnation au dessus de $35 \%$ dans la seconde moitié des années 1970 et les années 1980, le taux d'accès à l'enseignement supérieur a rapidement augmenté dans les années 1990, s'élevant à plus de $50 \%$ dans les années 2000 (52,3\% en 2006). ${ }^{1}$

Depuis quelques décennies, notamment depuis les années 1990, l'État a procédé à nombre de réformes universitaires. Celles-ci incluent une série de déréglementations relatives au régime des enseignants, l'abolition de la définition des domaines d'enseignement en 1991, la désétatisation des universités nationales en 2004, et la mise en place d'un système d'accréditation institutionnelle la même année.

Non seulement ces réformes ont considérablement modifié les comportements du personnel et des étudiants, mais elles ont modifié en profondeur le fonctionnement et la gestion des universités, jusqu'à produire une remise en cause de leurs missions fondamentales.

1. Le taux d'accès ne dépend pas uniquement du nombre de place déterminé. Il est tributaire de nombreux facteurs. 


\section{L'AUTONOMIE : CONTENUS ET MODALITÉS}

\section{La société du savoir}

Depuis le milieu des années 1980, le gouvernement a étudié, au sein du Conseil national de la réforme de l'éducation, une réforme complète du système éducatif japonais. Il prévoyait notamment des changements sociaux tels que l'internationalisation et l'informatisation de la société et le développement de l'apprentissage tout au long de la vie. Le Conseil a établi plusieurs rapports recommandant au gouvernement la reconnaissance de la diversité individuelle et sa valorisation, le passage à un système d'apprentissage tout au long de la vie et l'adaptation du système éducatif à l'internationalisation et l'informatisation. En matière d'enseignement supérieur, le Conseil a recommandé sa diversification, une augmentation de la part du financement privé et la réorganisation et l'amélioration managériale de l'université. Même si le Conseil n'a pas utilisé l'expression "société du savoir», ses recommandations prévoyaient son arrivée imminente.

En 1987, le ministère chargé de l'éducation (Monbusho) a établi un Conseil de l'université comme organisme chargé de délibérer des aspects fondamentaux en enseignement supérieur. Durant les années 1990, le Conseil a recommandé de nombreuses mesures telles que l'assouplissement du cadre national des diplômes, le développement de l'enseignement post-dispensé au niveau du mastère et du doctorat, la déréglementation et l'amélioration de l'administration universitaire. Ces propositions ont ensuite été mises en œuvre par le ministère.

En 1998, le Conseil de l'université a soumis un rapport intitulé Une vision pour l'université $d u X X I^{e}$ siècle et des mesures de réforme pour l'avenir : université compétitive dans un environnement concurrentiel qui présentait les lignes directrices des politiques d'enseignement supérieur dans la perspective du $\mathrm{XXI}^{\mathrm{e}}$ siècle et recommandait notamment de diversifier les universités et de les mettre en concurrence, d'assouplir le règlement relatif à leur structure, d'établir une administration universitaire permettant la prise de décisions responsables, et d'établir un système d'évaluation à multiples dimensions.

En outre, les Politiques pour la réforme structurelle de l'université (université nationale) du $\mathrm{MEXT}^{2}$ de 2001 ont défini la future direction de la réforme, en vue de rendre les universités nationales plus dynamiques et mondialement concurrentielles. Elles ont stipulé :

- le regroupement et la consolidation des universités nationales;

- l'introduction de méthodes managériales de secteur privé;

2. Celui-ci a remplacé le Monbusho en 2001. 
- la mise en place d'un principe de concurrence basé sur l'évaluation externe. En 2004, les universités nationales ont été constituées en établissements autonomes de droit public, après un processus de regroupement ${ }^{3}$.

En 2003, le MEXT a encore déréglementé l'enseignement universitaire. Les universités publiques et privées n'ont plus besoin d'une autorisation ministérielle pour modifier la structure des composantes académiques de base si la modification n'entraîne pas un changement de filière des diplômes concernés par cette modification. L'année suivante, le ministère a mis en place un système d'accréditation, en s'alignant sur la réforme administrative qui met davantage l'accent sur l'évaluation a posteriori.

Les réformes que nous venons de voir allaient dans le sens d'un agrandissement de l'autonomie universitaire et d'un renforcement de l'évaluation a posteriori.

\section{L'autonomie pédagogique}

En 1991, le Monbusho a assoupli les normes régissant le contenu de l'enseignement universitaire, et significativement élargi le périmètre des responsabilités des universités en cette matière. Avant cette réforme, les matières enseignées dans les universités devaient être classifiées en quatre catégories : "culture générale», "enseignement spécialisé », "langues étrangères », et «éducation physique et à la santé». Le nombre d'unités de cours dispensés dans chaque catégorie était défini par un arrêté ministériel et toutes les universités organisaient leurs programmes d'enseignement en fonction de cette règle. Désormais, l'obtention de 124 crédits ou plus est la seule condition requise par les nouvelles normes pour la délivrance d'un diplôme.

Cette réforme envisageait de diversifier les enseignements universitaires afin qu'ils soient adaptés à un effectif étudiant de plus en plus divers. Durant les années 1990, la massification de l'enseignement supérieur a davantage progressé. Alors que le taux d'accès au supérieur était de 36,3\% en 1990, celui-ci a atteint $49,1 \%$ en 2000 et en 2005 , il a finalement dépassé $50 \%$, ligne délimitée par Martin Trow entre l'enseignement supérieur de masse et l'enseignement supérieur universel (Trow, 1974). Par ailleurs, dans les années 1990, un durcissement du marché du travail a engendré une plus grande difficulté à trouver un emploi chez les diplômés.

Vers la fin des années 1990, les universités ont mis en place nombre d'outils pédagogiques. Parmi ces outils, citons la formation pédagogique des enseignants, la présentation du contenu des programmes, la semestrialisation des cursus, un système des notes globales moyennes obtenues par les étudiants ${ }^{4}$, l'assistant à l'enseignement et l'évaluation des enseignements par les étudiants.

3. Le processus continue encore aujourd'hui.

4. NDLR: Grade points average (GPA). 
En outre, les formations immédiatement utiles dans le milieu professionnel, telles que l'informatique et l'anglais, ont été enrichies. Depuis les années 2000, l'État a mis en place de nombreux dispositifs de soutien aux universités pour faire avancer leurs initiatives.

D'autre part, le régime du personnel enseignant a été révisé à plusieurs reprises, prévoyant notamment un assouplissement de leur profil. Ces modifications incluent une loi sur l'emploi des enseignants étrangers dans les universités publiques, la flexibilisation des conditions d'embauche des enseignants pour diverses expertises en dehors du monde académique et la mise en place d'un régime de l'emploi à durée déterminée.

\section{L'autonomie institutionnelle}

En 2004, les universités nationales, qui étaient un service du MEXT, ont été constituées en établissements autonomes de droit public (institutions universitaires nationales : IUN) ${ }^{5}$. Ceux-ci jouissent d'une autonomie agrandie et sont censés développer leur stratégie pour un meilleur enseignement-recherche. Le budget est alloué à chaque établissement sous forme de crédits globalisés sans préciser l'usage, sur la base des objectifs à moyen terme (OMT) et du plan à moyen terme (PMT). Les droits de scolarité ne sont plus fixés par l'État, qui détermine désormais seulement les montants standards, par rapport auxquels les IUN sont autorisés à augmenter les droits de $10 \%$ au maximum.

La gestion de l'université a été rationalisée et centralisée autour du président, pour permettre une prise de décision plus rapide. Le président est toujours nommé par le ministre sur la base de la proposition de l'université, mais celle-ci est élaborée par un comité de sélection du président qui est composé d'une dizaine de membres internes et externes ${ }^{6}$. La participation des experts externes à l'administration universitaire a été institutionnalisée. Ils siègent au conseil directeur et au conseil administratif ${ }^{7}$. En outre, la moitié des membres du comité de sélection du président doivent être des personnes externes. D'autre part, les membres du personnel ne sont plus fonctionnaires mais employés de l'IUN, ce qui flexibilise la gestion du personnel permettant diverses activités des enseignants (collaboration avec des entreprises, notamment) et le recrutement du personnel académique et non académique qualifié, incluant des étrangers. La nomination des personnels a été transférée du

5. Pour plus de détails, voir Oba (2005).

6. Avant la réforme, la proposition était formulée par un conseil composé exclusivement d'enseignants internes, généralement sur la base du résultat du vote de l'ensemble des enseignants. Après 2004, si la plupart des universités nationales ont gardé le système de vote, elles ont le plus souvent restreint le vote à ceux qui sont concernés par l'administration universitaire.

7. Ce sont des organes de direction. En outre, il y a un conseil d'enseignement et de recherche composé uniquement de membres internes. Ces trois conseils sont tous placés sous l'autorité du président. Le conseil directeur est le décideur du niveau le plus élevé avant la prise de décision par le président. 
ministre aux présidents d'université. Chaque IUN peut, par exemple, définir sa propre politique salariale, et mettre des postes administratifs au concours.

En échange de l'élargissement de l'autonomie, un système d'évaluation a été mis en place pour effectuer une évaluation externe a posteriori. Les IUN seront institutionnellement évaluées par le comité d'évaluation du MEXT, après chaque période des OMT/PMT (six ans). Pendant cette période, les IUN présentent chaque année leur bilan des activités incluant un rapport sur les travaux d'auto-évaluation. En outre, les IUN seront évaluées par une commission relevant du ministère de la gestion publique et des affaires internes en tant qu'établissements de droit public. Cette évaluation ne sera effectuée qu'au bout de la période des OMT/PMT, mais ses résultats pourront fortement influencer une révision du système d'IUN.

Les effets de la réforme sur l'enseignement-recherche et surtout sur l'administration universitaire sont perceptibles. D'après les rapports annuels des années fiscales 2004 et 2005 du comité d'évaluation du ministère, toutes les IUN ont adopté une politique budgétaire renforçant le budget à la disposition du président. Plus de deux tiers des présidents disposent de postes ou budget de personnel. Par ailleurs, plus de la moitié des universités ont mis en place un dispositif pour vérifier la pertinence de l'utilisation des ressources. En outre, après leur autonomisation, la plupart des IUN ont mis en place ou commencé à étudier un système d'évaluation des enseignants. Par ailleurs, les universités ont privilégié des programmes franchissant les frontières disciplinaires afin de mieux répondre aux besoins de la société et de renforcer l'excellence scientifique. Dans nombre d'universités, une grande partie des moyens discrétionnaires du président ont été utilisés pour développer les démarches interdisciplinaires qui dépasseraient les structures existantes rassemblant des chercheurs de différentes composantes et parfois de l'extérieur. Cependant, ces effets sont inégaux et différenciés plutôt qu'universels. Certaines universités tirent le meilleur profit de la marge d'autonomie étendue, en maîtrisant leurs missions et stratégies, leurs moyens d'action et leur fonctionnement avec une forte participation du personnel et en collaboration avec la communauté locale, alors que d'autres universités sont toujours en phase de consultation interne pour effectivement mettre en application leur plan d'action. Dans d'autres universités, les directions exercent l'autorité sur les composantes académiques, causant une profonde scission entre celles-ci et la présidence. Parfois, des présidents réformateurs n'ont plus la confiance des membres du personnel et sont mis en minorité.

\section{Autonomie et accréditation des universités}

En 2004, le ministère a mis en place un système d'accréditation. Ce nouveau système envisage d'améliorer continuellement la qualité de l'enseignement et de la recherche, et de garantir la qualité de l'offre de formation 
universitaire sur le plan national et international. Toutes les universités doivent passer une accréditation institutionnelle tous les sept ans (tous les cinq ans pour les écoles professionnelles) dans une agence d'accréditation.

Les agences d'accréditation ne relèvent nécessairement pas de la fonction publique, mais elles doivent être agréées par le MEXT. Le ministère doit répondre favorablement à la demande d'agrément lorsque celle-ci répond aux critères prédéterminés. L'accréditation concerne non seulement la formation mais tous les aspects de l'université. Chaque institution choisit une agence d'accréditation qui lui semble convenir le plus à ses missions. Celle-ci procède à des activités d'évaluation en fonction de ses propres normes d'évaluation. Les résultats des évaluations par les agences d'accréditation ne seront pas directement liés à la répartition des moyens publics, mais ils seront rendus publics et rapportés au MEXT. Chaque institution est censée réviser ses activités et structures en place et entreprendre des mesures nécessaires le plus rapidement possible sur la base du rapport d'évaluation qui lui est remis. Si le ministère reconnaît des négligences graves dans une institution après l'évaluation, il peut lui adresser une recommandation ou un ordre de se conformer aux normes ministérielles.

Trois agences ont été agréées par le MEXT pour l'accréditation des universités, dont une publique (nationale) et deux privées. S’y ajoute une agence privée, chargée exclusivement d'accréditation d'universités à cycle court. Entre les années fiscales 2004 et 2006, les quatre agences ont procédé à 217 cas d'évaluation, concernant environ 18,6\% des institutions. Pour l'année fiscale 2004, 34 universités ont été évaluées par la JUAA, première agence agréée. Sur ces 34 universités, 32 ont été accréditées mais toutes ont reçu des conseils et 22 d'entre elles se sont vues octroyer une accréditation avec recommandations. Par ailleurs, deux demandes ont été suspendues pour cause de non-conformité aux normes d'évaluation, même si les demandeurs satisfaisaient aux Normes d'institution des universités du MEXT. L'année suivante, les 25 demandeurs d'accréditation à la JUAA ont tous reçu une accréditation, dont 10 avec recommandations. En outre, les trois autres agences d'accréditation ont commencé à fonctionner cette même année.

\section{LA MISE EN GUVRE DES RÉFORMES}

\section{Effets}

Les effets des réformes sont, comme nous l'avons déjà vu, multiples et présents dans divers domaines d'activités universitaires. Mais ils sont très inégaux entre les établissements comme le montre le cas de l'acquisition des ressources externes par les IUN : selon les comptes financiers des IUN en 2004-2005, les dix meilleures universités sur 87 en ont remporté plus de $60 \%$. La mise en concurrence des universités est parfois favorable exclusivement pour les établissements se situant en haut de la hiérarchie, tels que les anciennes universités 
impériales. En outre, les réformes dans certains domaines, tels que l'enseignement, sont le plus souvent difficiles à réaliser. Afin d'avoir des résultats le plus rapidement possible, les décideurs concentrent parfois leurs efforts sur les domaines où ils peuvent avoir des conséquences rapides et faciles à démontrer, délaissant parfois les domaines de plus d'importance.

Néanmoins, l'enseignement supérieur japonais s'est sensiblement transformé ces dernières années. Les déréglementations relatives au régime des enseignants ont considérablement modifié le profil des enseignants du supérieur, et finalement fait disparaître le système des chaires professorales hérité du modèle humboldtien introduit au XIX ${ }^{\mathrm{e}}$ siècle. D’après des enquêtes auprès des enseignants universitaires, entre 1989 et 2003, la proportion des enseignants accordant de l'importance à l'enseignement est passée de $36 \%$ à $60 \%$ (RIHE, 2004). En outre, le pourcentage d'enseignants étrangers est passé de 1,3\% en 1985 à $3,5 \%$ en 2005. Par ailleurs, comme nous l'avons vu, des systèmes d'évaluation des enseignants se développent rapidement au sein des IUN.

Dans chaque université, de multiples outils pédagogiques ainsi que les soutiens aux étudiants ont été développés. Certaines universités ont regroupé les efforts pédagogiques en établissant divers centres au sein de l'université, tels que des centres d'études de la formation universitaire. Ces centres d'universités nationales travaillent collectivement dans le cadre d'une association : en juin 2007, trente centres y sont associés et collaborent pour une meilleure formation universitaire. Par ailleurs, nombre d'universités ont développé divers centres en vue de services aux étudiants, incluant les centres polyvalents de services et de soutien aux étudiants et les centres de l'orientation professionnelle. Dans ces centres, divers spécialistes ont été recrutés qui portent parfois le titre d'enseignant, en brouillant la distinction traditionnelle entre le personnel enseignant (personnel académique) et le personnel non enseignant (personnel administratif) du fait que ces spécialistes ne s'engagent guère dans des activités d'enseignement et de recherche dites disciplinaires ${ }^{8}$.

En matière de recherche, les approches interdisciplinaires et la coopération avec l'industrie ont été promues. Les universités ont amorcé des activités génératrices de revenu, incluant la coopération industrie/académie, l'industrialisation des résultats de la recherche, l'utilisation des propriétés intellectuelles, et l'appel à donation. Le nombre d'entreprises créées à partir d'activités des universités nationales est passé de 143 en 2001 à 738 en 2005, et la somme provenant des recherches conjointes et contractuelles a presque triplé (JANU, 2006). La plupart des universités nationales ont installé un bureau ou une société de transfert de technologie pour gérer les propriétés intellectuelles créées à l'université.

8. Ce phénomène s'observe dans les systèmes universitaires du monde face aux complications des tâches requises des universités (Whitchurch, 2004 ; Lombardi et al., 2002). 


\section{Défis majeurs}

Les réformes ont donné aux universités un nouveau cadre de fonctionnement plus souple. Cela doit leur permettre d'être plus efficaces et plus responsables en termes de programmes d'enseignement et de gestion des finances, des ressources humaines et du patrimoine, et de prendre des initiatives. Toutefois, les universités doivent assumer cette nouvelle responsabilité dans un environnement moins stable et plus compétitif entre les établissements, où le financement public décroît et où la population jeune est en diminution.

En matière de financement, l'État réduit chaque année la subvention de fonctionnement des IUN de $1 \%$ depuis la désétatisation, excepté la part de ces crédits correspondant à la masse salariale des enseignants. ${ }^{9}$ En mai 2006, une Loi pour la promotion des réformes administratives a été adoptée, stipulant que le salaire du personnel du gouvernement, incluant celui des IUN, devait être réduit de $5 \%$ avant 2010. En juin 2007, le ministère des Finances a déterminé les lignes directrices pour la préparation du budget 2008, et précisé que les modalités d'allocation de la subvention de fonctionnement des IUN devaient être révisées, en présentant un calcul préliminaire de celle-ci pour chaque IUN, sur la base d'un modèle compétitif, en fonction de l'acquisition des Subventions à la recherche scientifique des MEXT (Kakenhi). Selon lui, seulement treize universités augmenteraient leur subvention tandis que les autres (74) verraient leur subvention diminuer (dans certains cas, à plus de $80 \%$ ). Par ailleurs, les lignes directrices du ministère des Finances demandent la révision du système de bourses et de la subvention pour les universités privées.

L'évolution de la subvention de fonctionnement des IUN (en milliards de yen)

\begin{tabular}{|l|c|c|c|}
\hline Année fiscale & $\begin{array}{c}\text { Dotation pour } \\
\text { enseignement et recherche } \\
\text { (financement non compétitif) }\end{array}$ & $\begin{array}{c}\text { Dotation spéciale pour } \\
\text { enseignement et recherche } \\
\text { (financement compétitif) }\end{array}$ & $\begin{array}{c}\text { Prime de départ } \\
\text { à la retraite, etc. }\end{array}$ \\
\hline 2004 & 1036,9 & 74,1 & 130,5 \\
\hline 2005 & 1014,8 & 78,6 & 138,3 \\
\hline 2006 & 998,4 & 80,0 & 143,1 \\
\hline 2007 & 982,1 & 84,5 & 137,8 \\
\hline
\end{tabular}

Non seulement ces politiques déstabilisent le système d'enseignant supérieur, mais elles menacent l'égalité d'accès aux études supérieures. Les montants standards des droits de scolarité annuels ont été augmentés de 520800 yen ${ }^{10}$

9. Toutefois, chaque IUN n'est pas tenue de dépenser cette partie de ses recettes pour le salaire des enseignants. Elle peut librement décider du système de rémunération du personnel.

10. Un euro $=160$ yen. 
(3 255 euros) à 535800 yen (3 349 euros) en $2005^{11}$, et leur augmentation a encore été proposée par le ministère des Finances pour $2007^{12}$. En outre, lesdites lignes directrices du ministère des Finances recommandent une modulation des droits en fonction du contenu et de la qualité de la formation offerte. Ces politiques vont dans le sens contraire de l'égalité d'accès à l'enseignement supérieur, à laquelle ont largement contribué les universités nationales. Malgré la révision de 2005, certaines IUN ont bloqué, parfois partiellement, les droits de scolarité.

Par ailleurs, les réformes se sont inscrites dans un contexte de baisse des cohortes d'étudiants. Alors que le nombre de jeunes de dix-huit ans s'élevait à 2,05 millions en 1992, il n'était plus que de 1,30 million en 2007 et devrait atteindre 1,22 million en 2010, soit une baisse de $40 \%$. Malgré la progression du taux d'accès au supérieur, le nombre d'entrants au supérieur a commencé à diminuer depuis 1994. Cette diminution a, pourtant, été compensée par une prolongation des études, notamment par la conversion d'universités à cycle court en universités ordinaires et le développement des écoles "post-graduées». Toutefois, la compétition entre établissements pour attirer le plus grand nombre de meilleurs étudiants s'est intensifiée depuis ces dernières années, si bien que certains établissements ont même dû cesser leur activité faute de candidats.

Les autres défis sont multiples. Nombre d'IUN n'ont pas encore maîtrisé leur gestion et la collaboration entre elles dans ce domaine reste encore sous-développée. Le développement du personnel - académique et non académique - et la participation du personnel et des étudiants à la vie universitaire doivent aussi s'adapter à une gestion universitaire autonome. Les systèmes d'évaluation sont encore, pour la plupart, dans une phase d'essai ou d'étude. Surtout, l'auto-évaluation demeure peu ancrée dans les comportements et dans les pratiques de l'institution.

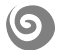

Depuis les années 1980, l'enseignement supérieur a fait l'objet de réformes dans nombre de pays du monde. Les réformes, liées aux profonds changements des objectifs de l'enseignement supérieur, allaient en général dans le sens d'un renforcement de l'autonomie universitaire et de l'évaluation (OCDE, 2003). Ce changement a été amorcé par la complexité de plus en plus grande de l'environnement dans lequel évoluent les universités (Eckel et Kezar, 2006), qui fait qu'il n'est plus opportun que les pouvoirs publics les gèrent directement. Les réformes universitaires au Japon, amorcées dans les années 1980 et accélérées dans les années 1990, s’alignaient grosso modo sur telles réformes.

11. Par ailleurs, les étudiants doivent s'acquitter de droits d'inscription dont le montant standard est fixé à 282000 yen ( 1763 euros) lors de l'inscription universitaire.

12. La proposition a finalement été retirée devant l'opposition des universités nationales et du MEXT, mais il a été décidé que les IUN pourraient augmenter leurs droits de $20 \%$ maximum à partir de la prochaine période des OMT/PMT. 
Comme nous l'avons vu, les réformes japonaises ont eu des impacts significatifs sur l'ensemble du système d'enseignement supérieur, incluant le régime et les comportements des enseignants, les modes de gestion de l'université, les modalités d'allocation du financement, et les systèmes d'évaluation. Toutefois, les défis sont multiples, et surtout l'environnement politico-administratif ne favorise pas une stabilisation du système universitaire. Par ailleurs, comme le montrent les orientations gouvernementales en matière de politiques économiques et financières validées en juin 2007, l'État envisage de développer certains pôles d'excellence universitaires, en diversifiant davantage les universités à travers la concurrence. L'égalité de l'accès aux études supérieures devrait en retour constituer un enjeu politique majeur.

Ce nonobstant, dans la société du savoir, les besoins de compétences se multiplient et l'enseignement supérieur doit demeurer au cœur de tous les débats relatifs au développement de ces compétences. Par ailleurs, comme le montre la Stratégie de Lisbonne, l'enseignement supérieur constitue un élément clé pour le développement économique et l'innovation. Les universités doivent répondre à ces besoins en effectuant des choix stratégiques dans la marge d'autonomie croissante. L'État doit, en plus de la mise en place d'un cadre réglementaire, apporter son soutien à la prise d'initiatives des universités, tout en s'en tenant à distance.

\section{Biblographie}

ECKEL P.D. and KEZAR A. (2006): "The Challenges Facing Academic Decision Making: Contemporary Issues and Steadfast Structures", in The Shifting Frontiers of Academic Decision Making: Responding to New Priorities, Following New Pathways. Eckel P.D., Praeger, Westport, 1-14.

JANU = Japan Association of National Universities (2006) : JANU Quarterly, hiver 2006, vol. 6, Author, Tokyo.

LOMBARDI J.V., et al. (2002) : University Organization, Governance, and Competitiveness. TheCenter, Gainesville.

OBA J. (2005): La constitution en société de l'université nationale au Japon Premières réactions des nouvelles organisations universitaires. Politiques et gestion de l'enseignement supérieur, vol. 17, $\mathrm{n}^{\circ} 2,119-139$.

OCDE = Organisation de coopération et de développement économique (2003): «Évolution des modes de gouvernance dans l'enseignement supérieur» in Analyse de politiques d'éducation. Éditions OCDE, Paris, 65-87.

RIHE $=$ Research Institute for Higher Education (2004): Study of the Institutionalization of Faculty Development Part 2: Report of the Nationwide Survey on University Faculty in 2003. COE Publication Series $n^{\circ} 10$, RIHE, Hiroshima.

TROW M. (1974): Problems in the Transition from Elite to Mass Higher Education. Policies for Higher Education. OECD, Paris, 51-101.

WHITCHURCH C. (2004): "Administrative Managers - A Critical Link". Higher Education Quarterly, vol. 58, nº (octobre 2004), 280-298. 